\title{
Editorial: Special issue on coding and cryptography
}

\author{
Faina Solov'eva ${ }^{1} \cdot$ Daniel Augot ${ }^{2} \cdot$ Thomas Johansson $^{3} \cdot$ Marine Minier $^{4}$. \\ Victor Zinoviev ${ }^{5}$
}

Published online: 12 January 2019

(c) Springer Science+Business Media, LLC, part of Springer Nature 2019

This volume contains 33 thoroughly refereed contributions concerning a wide range of topics in coding and cryptography. Almost all selected papers are the full journal versions of extended abstracts accepted for presentation at the International Workshop on Coding and Cryptography (WCC 2017) held in Saint Petersburg, Russia, September 18-22, 2017.

The aim of WCC is to bring together researchers in all aspects of coding theory, cryptology and related areas, theoretical or applied. Each of the 75 extended abstracts originally submitted to the workshop were reviewed by at least two members of the Program Committee. As a result of the screening process, 48 papers were selected for the presentation at the workshop on Coding and Cryptography together with five invited talks, by Alexander Barg, Claude Carlet, Camilla Hollanti, Grigory A. Kabatiansky and Patrick R. J. Östergård.

The papers presented at the workshop reflect the variety of themes involving both coding and cryptography, following the now well established tradition of the workshop. The authors of the presented papers were invited to submit full versions of their papers to the journal Designs, Codes and Cryptography. Each of the full-version submissions were once again

$\bowtie \quad$ Daniel Augot

Daniel.Augot@inria.fr

Faina Solov'eva

sol@math.nsc.ru

Thomas Johansson

thomas.johansson@eit.lth.se

Marine Minier

marine.minier@loria.fr

Victor Zinoviev

zinov@iitp.ru

1 Sobolev Institute of Mathematics and the Novosibirsk State University, 4 Acad. Koptyug avenue, 630090 Novosibirsk, Russia

2 INRIA, 1 rue d'Estienne d'Orves, campus de l'École polytechnique, 91120 Palaiseau, France

3 Lund University, Paradisgatan 2, Lund SE-221 00, Sweden

4 Université de Lorraine, LORIA Laboratory, Campus Scientifique, BP 239, 54506 Vandoeuvre-lès-Nancy, France

5 Institute for Information Transmission Problems, Russian Academy of Sciences, Bol'shoi Karetnyi per. 19, GSP-4, 127994 Moscow, Russia 
thoroughly refereed by at least two anonymous reviewers. This volume is the end result of this full-fledged reviewing process.

We are very grateful to all reviewers of these papers. We would like to thank all the authors and all the other participants of the WCC2017 for making it a highly stimulating meeting.

Publisher's Note Springer Nature remains neutral with regard to jurisdictional claims in published maps and institutional affiliations. 